\title{
Caracterização e Impacto Clínico Tardio do No-Reflow Associado a Intervenção Coronária Percutânea Primária vs. Eletiva
}

\author{
Carlos Augusto Homem de Magalhães Campos', Henrique Ribeiro', Expedito E. Ribeiro', \\ Andre G. Spadaro' ${ }^{1}$ Pedro A. Lemos ${ }^{1}$, Marco Perin ${ }^{1}$, Gilberto Marchiori ${ }^{1}$, Pedro Horta ${ }^{1}$, \\ Luiz J. Kajita', Eulogio Martinez ${ }^{1}$, José Antonio F. Ramires ${ }^{1}$
}

\section{RESUMO}

Introdução: Apesar dos grandes avanços alcançados pela cardiologia intervencionista, o fenômeno do no-reflow ainda ocorre durante as intervenções coronárias percutâneas (ICP) e está associado a pior prognóstico. O objetivo deste estudo foi caracterizar os perfis clínico, angiográfico e do procedimento de pacientes com no-reflow, além de avaliar seu impacto clínico tardio. Método: Analisamos, no período de janeiro de 2004 a fevereiro de 2009, todos os pacientes submetidos a ICP no Instituto do Coração (InCor), que em qualquer momento da intervenção apresentaram no-reflow, mesmo que transitoriamente. Os pacientes foram divididos em dois grupos: no-reflow de reperfusão (associado a ICP primária) e no-reflow de intervenção (associado a ICP eletiva). A probabilidade de óbito foi estimada pelo método de Kaplan-Meier e a regressão de Cox foi utilizada para identificar seus preditores. Resultados: Foram avaliados 132 pacientes consecutivos, 81 no grupo no-reflow de reperfusão e 51 no grupo no-reflow de intervenção. O sucesso do procedimento foi obtido em $83,5 \%$ da população total $(80,2 \%$ vs. $90,2 \%$, respectivamente; $P=0,149)$. A probabilidade de mortalidade tardia foi estimada em 38,6\%, sendo maior no grupo de no-reflow de reperfusão $(55,8 \%$ vs. $11,1 \% ; P=0,005)$. Na análise multivariada, somente o sexo feminino [hazard ratio (HR) 2,5, intervalo de confiança de $95 \%$ (IC 95\%) 1,22-5,14; P = 0,027) e a doença pulmonar obstrutiva crônica (HR 9,3, IC 95\% 1,45-60,14; P = 0,027) foram preditores independentes de mortalidade, enquanto o uso prévio de estatina foi um fator de proteção (HR 0,15, IC 95\% 0,05-0,48; P = 0,002). Conclusão: $O$ fenômeno de no-reflow foi associado a elevados índices de insucesso

\section{ABSTRACT}

Characterization and Late Clinical Impact of No-Reflow Associated to Primary Percutaneous Coronary Intervention vs. Elective Percutaneous Coronary Intervention

Background: Despite major advances in interventional cardiology in recent years, no-reflow is still observed during percutaneous coronary interventions $(\mathrm{PCl})$, and is associated to a worse prognosis. The objective of this study was to characterize the clinical, angiographic and procedural profile of patients with no-reflow as well as assessing its late clinical impact. Method: From January 2004 to February 2009, patients undergoing $\mathrm{PCl}$ at the Instituto do Coração (InCor), presenting no-reflow at any time during the intervention, were evaluated. Patients were divided into two groups: reperfusion no-reflow (associated to primary $\mathrm{PCl}$ ) and interventional no-reflow (associated to elective $\mathrm{PCI}$ ). The probability of death was estimated by the Kaplan-Meier method and Cox regression was used to identify its predictors. Results: One hundred and thirty-two consecutive patients were evaluated, 81 in the reperfusion no-reflow group and 51 in the interventional no-reflow group. Procedural success was observed in $83.5 \%$ of the overall population $(80.2 \%$ vs. $90.2 \%$, respectively; $\mathrm{P}=0.149)$. The long-term probability of death was estimated at $38.6 \%$, and was higher in the reperfusion no-reflow group $(55.8 \%$ vs. $11.1 \%$; $\mathrm{P}=0.005)$. In the multivariate analysis, only female gender [hazard ratio (HR) 2.5, 95\% confidence interval $(95 \% \mathrm{Cl}) 1.22$ $5.14 ; \mathrm{P}=0.027)$ and chronic obstructive pulmonary disease (HR 9.35, Cl 95\% 1.45-60.14; $\mathrm{P}=0.027)$ were independent predictors of mortality, whereas the prior use of statin was

1 Instituto do Coração do Hospital das Clínicas da Faculdade de Medicina da Universidade de São Paulo (InCor/HCFMUSP) - São Paulo, SP, Brasil.

Correspondência: Carlos Augusto Homem de Magalhães Campos. Av. Dr. Enéas Carvalho de Aguiar, 255 - Cerqueira César - São Paulo, SP, Brasil - CEP 05403-000

E-mail:cm-campos@uol.com.br

Recebido em: 14/7/2010 • Aceito em: 27/8/2010 
Campos CAHM, et al. Caracterização e Impacto Clínico Tardio do No-Reflow Associado a Intervenção Coronária Percutânea Primária vs. Eletiva. Rev Bras Cardiol Invasiva. 2010;18(3):300-5.

do procedimento e mortalidade tardia, principalmente quando associado a angioplastia primária.

DESCRITORES: Mortalidade. Infarto do miocárdio. Angioplastia transluminal percutânea coronária. Reperfusão.

A intervenção coronária percutânea é um procedimento bem estabelecido na terapêutica da doença arterial coronária, mas ocasionalmente seu resultado é comprometido pela ocorrência do noreflow. ${ }^{1,2}$ Esse fenômeno, caracterizado pelo estado de hipoperfusão do tecido miocárdio na presença de uma artéria coronária epicárdica patente, é classificado de acordo com a duração da isquemia miocárdica precedente. ${ }^{3}$ É chamado de no-reflow de reperfusão quando ocorre durante a intervenção coronária percutânea primária no infarto agudo do miocárdio, após a recanalização da artéria culpada, podendo ser assintomático ou apresentar-se clinicamente como dor torácica contínua e persistência da elevação do segmento ST. Há também o no-reflow de intervenção, que acontece na intervenção coronária percutânea eletiva, em indivíduos que não estiveram expostos a isquemia prolongada antes da intervenção. Em geral, é de início súbito e cursa com isquemia aguda e alterações eletrocardiográficas. ${ }^{4}$ Nos dois cenários, a ocorrência desse fenômeno está associada a pior prognóstico. O fluxo coronário reduzido (fluxo TIMI 0 a 2) após intervenção coronária percutânea primária está relacionado à ocorrência de insuficiência cardíaca e maior mortalidade, independentemente do tamanho do infarto. ${ }^{5} \mathrm{O}$ no-reflow de intervenção está associado a maiores índices de infarto e óbito após intervenção coronária percutânea. ${ }^{6}$ A caracterização tanto clínica como angiográfica e do procedimento das duas formas, entretanto, ainda não está bem estabelecida. O objetivo deste estudo, portanto, foi comparar o perfil dos pacientes nos grupos no-reflow de reperfusão e de intervenção e avaliar seus desfechos clínicos tardios.

\section{MÉTODOS}

\section{População do estudo}

Os pacientes submetidos a intervenção coronária percutânea no Instituto do Coração do Hospital das Clínicas da Faculdade de Medicina da Universidade de São Paulo (InCor/HCFMUSP), no período de janeiro de 2004 a fevereiro de 2009, foram avaliados, tendo sido incluídos os indivíduos que, em algum momento da intervenção, apresentaram, mesmo que transitoriamente, quadro de no-reflow. Para avaliarmos as características clínicas, angiográficas e do procedimento, bem como o impacto do tipo de no-reflow na evolução tardia, dividimos essa população em dois grupos: a protective factor $(\mathrm{HR} 0.15,95 \% \mathrm{Cl} 0.05-0.48 ; \mathrm{P}=0.002)$. Conclusion: The no-reflow phenomenon was associated with high procedural failure rates and long-term mortality, especially when associated to primary angioplasty.

KEY-WORDS: Mortality. Myocardial infarction. Angioplasty, transluminal, percutaneous coronary. Reperfusion.

- no-reflow de reperfusão $(\mathrm{n}=81)$, que incluiu pacientes submetidos a intervenção coronária percutânea primária no infarto agudo do miocárdio com supradesnível do segmento ST; e

- no-reflow de intervenção $(n=51)$, que incluiu pacientes submetidos a intervenção coronária percutânea eletiva nas síndromes coronárias agudas sem supradesnível do segmento ST ou angina estável.

Os dados da evolução intra-hospitalar foram coletados por médicos treinados, durante a internação índice, seguindo o preenchimento de formulários previamente padronizados. A coleta incluiu características clínicas, resultados de exames laboratoriais, dados do procedimento invasivo e evolução clínica até a alta hospitalar. Após a alta, os pacientes foram periodicamente avaliados em nosso ambulatório. A ocorrência de óbito foi avaliada por meio da revisão de prontuários ou por contato telefônico, quando necessário.

\section{Definições}

O diagnóstico de infarto agudo do miocárdio com supradesnível do segmento ST foi realizado na ocorrência de elevação persistente de ST maior que $1 \mathrm{~mm}$ em duas derivações contíguas ou novo bloqueio de ramo esquerdo ao eletrocardiograma. Angina estável foi considerada quando havia dor desencadeada por esforço, isquemia silenciosa documentada por prova funcional ou equivalente anginoso. Angina instável foi definida como dor em repouso, de início recente ou progressiva, sem os critérios eletrocardiográficos citados anteriormente; quando associada a elevação de marcadores de necrose miocárdica, foi considerada infarto do miocárdio sem supradesnível do segmento ST. Presença de trombo à angiografia foi definida como interrupção abrupta no fluxo do vaso com retenção do contraste ou falha de enchimento em vaso patente ("imagem negativa"), sobre ou adjacente a uma região estenótica. Sucesso angiográfico foi definido por redução da lesão-alvo < 30\%, com manutenção ou restabelecimento do fluxo anterógrado normal (TIMI 3). ${ }^{7}$

\section{Análise estatística}

Variáveis categóricas foram apresentadas como frequências e porcentagens e comparadas com teste de qui-quadrado. As variáveis contínuas foram descritas como médias e desvios padrão ou medianas e intervalo interquartil (percentis 25 e 75) e comparadas 
com o teste de Wilcoxon. A probabilidade de óbito foi estimada pelo método de Kaplan-Meier. Modelos univariados e multivariados, utilizando a regressão de Cox, foram utilizados para identificar a relação entre possíveis preditores (variáveis apresentadas nas Tabelas 1 e 2) e mortalidade durante o seguimento clínico. Todos os testes foram bicaudais e um valor de $\mathrm{P}<0,05$ foi considerado significativo.

\section{RESULTADOS}

Foram incluídos 132 pacientes consecutivos nesta análise, com mediana de seguimento de 449 dias (intervalo interquartil de 188-706 dias). A média de idade foi de $64,2 \pm 9,7$ anos, sendo $75,9 \%$ do sexo masculino, 28,6\% de diabéticos, 15\% com insuficiência cardíaca e 70,7\% com padrão coronário multiarterial. Na comparação entre os grupos, conforme demonstrado na Tabela 1, os pacientes apresentaram perfil clínico similar, exceto pela maior ocorrência, no grupo no-reflow de intervenção, de dislipidemia (46,4\% vs. $82,4 \%$; $P<0,001$ ), infarto prévio ( $25 \%$ vs. $54,9 \%$; $P<0,001)$ e cirurgia de revascularização prévia $(7,8 \%$ vs. $41,2 \% ; P<0,001)$. No que tange ao tratamento medicamentoso empregado previamente à intervenção, pudemos observar, nesse grupo, maior utilização de estatinas $(66,7 \%$ vs. 95,2\%; P $<0,001)$ e de inibidores de glicoproteína Ilb/IIla (20\% vs. 59,5\%; P < 0,001).

Na Tabela 2, que apresenta a comparação das características angiográficas, observam-se semelhanças entre os grupos em relação ao tamanho dos stents utilizados (diâmetro e extensão), bem como ao número total de lesões abordadas por paciente e ao volume de contraste utilizado. O grupo no-reflow de reperfusão demonstrou mais frequentemente a presença de trombo identificável à angiografia (55,6\% vs. 19,6\%; P<0,001). Observamos diferentes comportamentos entre os grupos no que diz respeito ao território tratado e à abordagem de enxertos venosos.

\section{Desfechos clínicos}

Sucesso do procedimento foi obtido em 83,5\% da população total, sendo numericamente inferior no grupo no-reflow de reperfusão, embora sem alcançar diferença estatística $(80,2 \%$ vs. 90,2\%; P = 0,149).

A mortalidade estimada aos 449 dias (mediana do tempo de seguimento) foi de $38,6 \%$, sendo maior para o grupo no-reflow de reperfusão que para o grupo noreflow de intervenção $(55,8 \%$ vs. $11,1 \% ; P=0,005)$ (Figura 1).

TABELA 1

Características clínicas

\begin{tabular}{|c|c|c|c|}
\hline & $\begin{array}{c}\text { No-reflow } \\
\text { de reperfusão }(n=81)\end{array}$ & $\begin{array}{c}\text { No-reflow } \\
\text { de intervenção }(n=51)\end{array}$ & $\mathbf{P}$ \\
\hline Idade, anos & $64,3 \pm 10$ & $64,1 \pm 9$ & 0,70 \\
\hline Sexo masculino, \% & 75 & 80,4 & 0,52 \\
\hline Diabetes melito, \% & 23,5 & 35,3 & 0,16 \\
\hline Hipertensão arterial sistêmica, \% & 77,6 & 74,5 & 0,67 \\
\hline Dislipidemia, \% & 46,4 & 82,4 & $<0,001$ \\
\hline Tabagismo, \% & 30,3 & 20 & 0,22 \\
\hline AVC prévio, \% & 6,6 & 2 & 0,4 \\
\hline DPOC, \% & 3,9 & 4 & $>0,9$ \\
\hline IRC dialítica, \% & 2,6 & 0 & 0,51 \\
\hline IVP, \% & 1,3 & 4 & 0,56 \\
\hline IAM prévio, \% & 25 & 54,9 & 0,001 \\
\hline Insuficiência cardíaca, \% & 13,5 & 20 & 0,46 \\
\hline ICP prévia, \% & 10,7 & 17,6 & 0,29 \\
\hline CRM prévia, \% & 7,8 & 41,2 & $<0,001$ \\
\hline \multicolumn{4}{|l|}{ Medicações pré-intervenção, \% } \\
\hline Ácido acetilsalicílico & 98,7 & 100 & 0,9 \\
\hline Clopidogrel & 36,7 & 40,5 & 0,83 \\
\hline Estatinas & 66,7 & 95,2 & $<0,001$ \\
\hline Inibidor de glicoproteína IIb/IIla & 20 & 59,5 & $<0,001$ \\
\hline
\end{tabular}

$\overline{\mathrm{AVC}}=$ acidente vascular cerebral, $\mathrm{CRM}=$ cirurgia de revascularização miocárdica; DPOC = doença pulmonar obstrutiva crônica; IAM = infarto agudo do miocárdio; ICP = intervenção coronária percutânea; IRC = insuficiência renal crônica; IVP = insuficiência vascular periférica; $\mathrm{n}=$ número de pacientes. 
TABELA 2

Características angiográficas e do procedimento

\begin{tabular}{|c|c|c|c|}
\hline & $\begin{array}{c}\text { No-reflow } \\
\text { de reperfusão }(\mathrm{n}=\mathbf{8 1})\end{array}$ & $\begin{array}{c}\text { No-reflow } \\
\text { de intervenção }(n=51)\end{array}$ & $\mathbf{P}$ \\
\hline Padrão de acometimento coronário, \% & & & 0,08 \\
\hline Uniarterial & 34,6 & 20 & \\
\hline Multiarterial & 65,4 & 80 & \\
\hline Vaso tratado, \% & & & $<0,001$ \\
\hline Enxerto venoso & 8,6 & 39,2 & \\
\hline Artéria nativa & 91,4 & 60,8 & \\
\hline Território tratado, \% & & & 0,03 \\
\hline Descendente anterior & 48,1 & 27,5 & \\
\hline Circunflexa & 7,4 & 17,6 & \\
\hline Coronária direita & 44,4 & 54,9 & \\
\hline Presença de trombo, \% & 55,6 & 19,6 & $<0,001$ \\
\hline Diâmetro do stent, mm & $3,17 \pm 0,57$ & $3,28 \pm 0,67$ & 0,38 \\
\hline Extensão do stent, mm & $24,23 \pm 9,3$ & $23,28 \pm 7,7$ & 0,56 \\
\hline Total de lesões/paciente & $1,31 \pm 0,58$ & $1,41 \pm 0,66$ & 0,35 \\
\hline Volume de contraste utilizado, $\mathrm{ml}$ & $260,26 \pm 71,6$ & $277,7 \overline{6} \pm 89$ & 0,22 \\
\hline
\end{tabular}

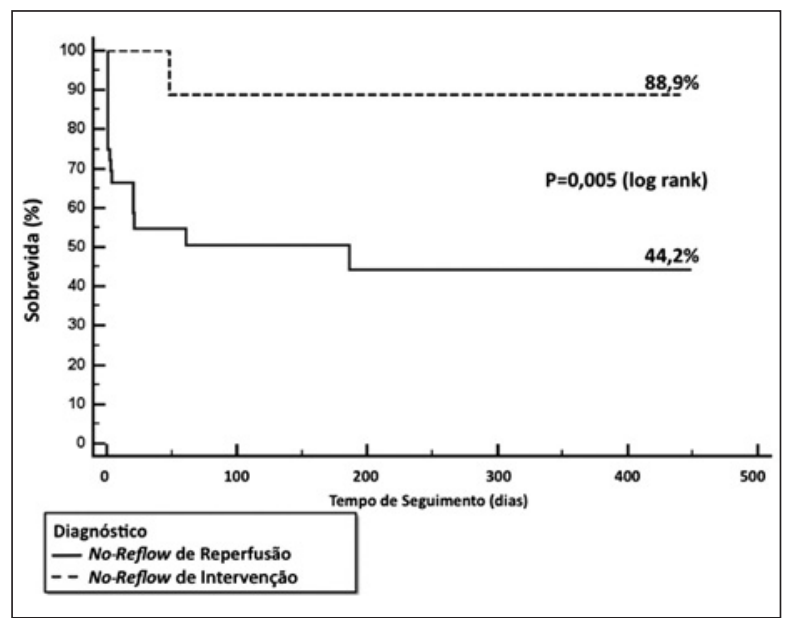

Figura 1 - Probabilidade de sobrevida tardia entre os pacientes com no-reflow de reperfusão e no-reflow de intervenção.

\section{Preditores de mortalidade tardia}

À análise univariada, foram identificados os seguintes preditores de mortalidade: insucesso do procedimento, sexo feminino, presença de doença pulmonar obstrutiva crônica e insuficiência cardíaca. O uso de estatinas exerceu efeito protetor para a sobrevida tardia. Quando os dados foram inseridos em um ajuste multivariado (Tabela 3), somente sexo feminino e doença pulmonar obstrutiva crônica permaneceram como preditores independentes de mortalidade. O benefício das estatinas permaneceu mesmo após análise multivariada.
TABELA 3

Variáveis que interferiram independentemente na mortalidade tardia

\begin{tabular}{lcc}
\hline Variável & $\begin{array}{c}\text { Hazard ratio } \\
\text { ajustado (IC 95\%) }\end{array}$ & $\mathbf{P}$ \\
\hline DPOC & $9,3(1,45-60,14)$ & 0,027 \\
Sexo feminino & $2,5(1,22-5,14)$ & 0,027 \\
Uso prévio de estatinas & $0,15(0,05-0,48)$ & 0,002
\end{tabular}

DPOC = doença pulmonar obstrutiva crônica; IC 95\% = intervalo de confiança de $95 \%$.

\section{DISCUSSÃO}

Os principais achados deste estudo foram: (1) o no-reflow esteve associado a elevados índices de insucesso do procedimento $(16,5 \%)$ e a probabilidade de mortalidade tardia $(38,6 \%)$; (2) o no-reflow de reperfusão e o no-reflow de intervenção apresentaram características clínicas e angiográficas distintas; (3) pior prognóstico no grupo de no-reflow associado a intervenção coronária percutânea primária; (4) sexo feminino, doença pulmonar obstrutiva crônica e estatinas foram características que modificaram a evolução clínica dos pacientes.

Estudos prévios demonstraram que a ocorrência de no-reflow é uma complicação infrequente, mas de grave repercussão clínica. Abbo et al. ${ }^{8}$ analisaram 10.676 intervenções coronárias, observando no-reflow em 0,6\% 
delas. No entanto, quando presente, essa complicação foi associada a incremento de 10 vezes na ocorrência de óbito e infarto quando comparada com a mortalidade de pacientes sem no-reflow. ${ }^{8}$ Da mesma forma, Ndrepepa et al. ${ }^{9}$ estimaram mortalidade de um ano de $16,7 \%$ na ocorrência de no-reflow e de 5,5\% na ausência deste. Nossos dados, oriundos de uma população brasileira, demonstram que, de fato, o no-reflow está associado a considerável mortalidade global tardia, sendo portanto comparáveis aos da literatura.

Pode-se inferir que a elevada mortalidade tardia esteja relacionada à não desprezível taxa de insucesso do procedimento, sendo esta de $16,5 \%$ na população total de nosso estudo. Em nossa análise, apesar de não alcançar significância estatística, o insucesso do procedimento foi o dobro no cenário da intervenção coronária percutânea primária quando comparado ao da intervenção coronária percutânea eletiva (19,8\% vs. $9,8 \% ; P=0,149)$. Essa tendência pode ter fundamento fisiopatológico. Muitas vezes, de forma simplista, a microembolização coronária é responsabilizada como causa do no-reflow e, apesar de contribuir nesse cenário clínico, não é a única responsável. Estudos experimentais demonstram que uma oclusão extrínseca temporária de vaso epicárdico normal pode resultar em no-reflow quando a artéria é liberada. O no-reflow fica restrito à região onde há dano capilar. ${ }^{10}$ Isso ocorre porque no mecanismo do no-reflow de reperfusão, além da embolização, estão incluídos danos teciduais secundários à isquemia prolongada, como injúria de reperfusão, edema miocárdico, dano capilar e resposta inflamatória. Já na fisiopatologia do no-reflow de intervenção estão descritos principalmente a obstrução microvascular e a resposta inflamatória como responsáveis pela interrupção do fluxo. ${ }^{4} \mathrm{Em}$ nossa população, em que todos os pacientes tiveram oclusão do vaso intraprocedimento, o diagnóstico de admissão teve efeito determinante na sobrevida tardia. No infarto agudo do miocárdio, a isquemia extensa e prolongada prévia à intervenção, que leva a comprometimento da rede capilar e extenso dano miocárdico, pode explicar a maior probabilidade de mortalidade tardia para o no-reflow de reperfusão $(55,8 \%$ vs. $11,1 \% ; P<0,001)$.

Ainda assim, mesmo com bases fisiopatológicas distintas, pudemos verificar forte influência da microembolização nesses eventos. Nos casos de no-reflow de intervenção, quase $40 \%$ das intervenções foram em enxertos venosos. As intervenções em pontes de safena podem ser desafiadoras justamente pelos elevados índices de embolização distal, levando a no-reflow em cerca de $15 \%$ das angioplastias. ${ }^{11}$ Dessa forma, fica evidente que nas intervenções eletivas a abordagem dos enxertos venosos é uma das grandes responsáveis pelo no-reflow. Outro reconhecido preditor de no-reflow é a presença de trombo, que no no-reflow de reperfusão foi identificado em mais da metade dos casos (55,6\%). No infarto agudo do miocárdio, a remoção de material trombótico e substâncias solúveis relacionadas ao trom- bo reduz a carga trombótica e melhora a perfusão miocárdica. ${ }^{12-15}$ Os achados de nosso estudo reforçam a importância de estratégias recomendadas pelas diretrizes vigentes, ao utilizar filtros de proteção nas intervenções de enxertos venosos (classe I, nível de evidência A) e tromboaspiração no infarto agudo do miocárdio com supradesnível do segmento ST (classe Ila, nível de evidência B). ${ }^{16}$

Os preditores independentes de mortalidade identificados foram presença de doença pulmonar obstrutiva crônica e sexo feminino, enquanto o uso de estatinas exerceu efeito protetor. Os resultados mais contemporâneos de intervenções coronárias percutâneas eletivas ou na vigência de infarto agudo do miocárdio, com implante ou não de stents farmacológicos, e após os ajustes para as variáveis de confusão erradicaram o rótulo de maior risco para procedimentos de intervenção em pacientes do sexo feminino, tanto nos resultados imediatos como na evolução tardia. ${ }^{16,17}$ Em nossa casuística, variáveis não mensuradas, como fragilidade física e menor superfície corporal, podem ter influenciado nossos resultados.

Por outro lado, as estatinas demonstraram estabilizar a placa aterosclerótica vulnerável, pela redução de seu conteúdo lipídico e por sua ação anti-inflamatória, que também reduz a resposta inflamatória miocárdica associada à microembolização coronária e a microinfartos. Dados da literatura demonstram que pacientes que recebem estatinas apresentam menor injúria miocárdica periprocedimento, identificada por menor liberação de creatina quinase fração $\mathrm{MB}(\mathrm{CK}-\mathrm{MB})$ e troponina, menor resposta inflamatória e melhor evolução a longo prazo. ${ }^{18-23} \mathrm{Em}$ nossa amostra, constituída por pacientes de alto risco, ficam evidentes os benefícios dessa classe de medicação na farmacologia adjunta da intervenção coronária percutânea.

\section{CONCLUSÃO}

O fenômeno de no-reflow foi associado a elevados índices de insucesso do procedimento e a mortalidade tardia, especialmente quando associado à angioplastia primária.

\section{CONFLITO DE INTERESSES}

Os autores declararam inexistência de conflito de interesses relacionado a este manuscrito.

\section{REFERÊNCIAS}

1. Morishima I, Sone T, Mokuno S, Taga S, Shimauchi A, Oki Y, et al. Clinical significance of no-reflow phenomenon observed on angiography after successful treatment of acute myocardial infarction with percutaneous transluminal coronary angioplasty. Am Heart J. 1995;130(2):239-43.

2. Gibson CM, Schömig A. Coronary and myocardial angiography: angiographic assessment of both epicardial and myocardial perfusion. Circulation. 2004;109(25):3096-105. 
3. Kloner RA, Ganote CE, Jennings RB. The "no-reflow" phenomenon after temporary coronary occlusion in the dog. J Clin Invest. 1974;54(6):1496-508.

4. Jaffe R, Charron T, Puley G, Dick A, Strauss BH. Microvascular obstruction and the no-reflow phenomenon after percutaneous coronary intervention. Circulation. 2008;117(24):3152-6.

5. Morishima I, Sone T, Okumura K, Tsuboi H, Kondo J, Mukawa $\mathrm{H}$, et al. Angiographic no-reflow phenomenon as a predictor of adverse long-term outcome in patients treated with percutaneous transluminal coronary angioplasty for first acute myocardial infarction. J Am Coll Cardiol. 2000;36(4):1202-9.

6. Abbo KM, Dooris M, Glazier S, O'Neill WW, Byrd D, Grines $\mathrm{CL}$, et al. Features and outcome of no-reflow after percutaneous coronary intervention. Am J Cardiol. 1995; 75(12):778-82.

7. TIMI Study Group. The Thrombolysis in Myocardial Infarction (TIMI) trial. Phase-I findings. N Engl J Med. 1985;312(14): 932-6.

8. Abbo KM, Dooris M, Glazier S, O'Neill WW, Byrd D, Grines $\mathrm{CL}$, et al. Features and outcome of no-reflow after percutaneous coronary intervention. Am J Cardiol. 1995;75(12): 778-82.

9. Ndrepepa G, Tiroch K, Keta D, Fusaro M, Seyfarth M, Pache J, et al. Predictive factors and impact of no reflow after primary percutaneous coronary intervention in patients with acute myocardial infarction. Circ Cardiovasc Interv. 2010; $3(1): 27-33$

10. Reffelmann T, Kloner RA. The no-reflow phenomenon: a basic mechanism of myocardial ischemia and reperfusion. Basic Res Cardiol. 2006;101(5):359-72.

11. Sdringola S, Assali AR, Ghani M, Moustapha A, Achour H, Yusuf SW, et al. Risk assessment of slow or no-reflow phenomenon in aortocoronary vein graft percutaneous intervention. Catheter Cardiovasc Interv. 2001;54(3):318-24.

12. Leineweber $K$, Böse $D$, Vogelsang $M$, Haude $M$, Erbel $R$, Heusch G. Intense vasoconstriction in response to aspirate from stented saphenous vein aortocoronary bypass grafts. J Am Coll Cardiol. 2006;47(5):981-6.

13. Amin AP, Mamtani MR, Kulkarni H. Factors influencing the benefit of adjunctive devices during percutaneous coronary intervention in ST-segment elevation myocardial infarction: meta-analysis and meta-regression. J Interv Cardiol. 2009; 22(1):49-60.

14. Svilaas T, Vlaar PJ, van der Horst IC, Diercks GF, de Smet
BJ, van den Heuvel AF, et al. Thrombus aspiration during primary percutaneous coronary intervention. $\mathrm{N}$ Engl J Med. 2008;358(6):557-67.

15. Vlaar PJ, Svilaas T, van der Horst IC, Diercks GF, Fokkema ML, de Smet BJ, et al. Cardiac death and reinfarction after 1 year in the Thrombus Aspiration during Percutaneous coronary intervention in Acute myocardial infarction Study (TAPAS): a 1-year follow-up study. Lancet. 2008;371(9628): 1915-20.

16. Mattos LA, Lemos Neto PA, Rassi Junior A, Marin-Neto JA, Sousa AGMR, Devito FS, et al. Diretrizes da Sociedade Brasileira de Cardiologia. Intervenção coronária percutânea e métodos adjuntos diagnósticos em cardiologia intervencionista. (II Edição - 2008). Arq Bras Cardiol. 2008;91(6 Supl 1):1-58.

17. Lansky AJ, Hochman JS, Ward PA, Mintz GS, Fabunmi R, Berger $\mathrm{PB}$, et al. Percutaneous coronary intervention and adjunctive pharmacotherapy in women: a statement for healthcare professionals from the American Heart Association. Circulation. 2005;111(7):940-53.

18. Herrmann J, Lerman A, Baumgart D, Volbracht L, Schulz R, von Birgelen $\mathrm{C}$, et al. Preprocedural statin medication reduces the extent of peri-procedural non-Q-wave myocardial infarction. Circulation. 2002;106(17):2180-3.

19. Chan AW, Bhatt DL, Chew DP, Quinn MJ, Moliterno DJ, Topol EJ, et al. Early and sustained survival benefit associated with statin therapy at the time of percutaneous coronary intervention. Circulation. 2002;105(6):691-6.

20. Mulukutla SR, Marroquin OC, Smith C, Varghese R, Anderson $W D$, Lee JS, et al. Effect of statin therapy prior to elective percutaneous coronary intervention on frequency of periprocedural myocardial injury. Am J Cardiol. 2004;94(11): 1363-6.

21. Briguori C, Colombo A, Airoldi F, Violante A, Focaccio A, Balestrieri $P$, et al. Statin administration before percutaneous coronary intervention: impact on periprocedural myocardial infarction. Eur Heart J. 2004;25(20):1822-8.

22. Veselka J, Prochazkova S, Duchonova R, Homolova I, Tesar D, Bybee KA. Preprocedural statin therapy reduces the risk and extent of cardiac biomarker release following percutaneous coronary intervention. Heart Vessels. 2006;21(3):146-51.

23. Auguadro C, Manfredi M, Scalise F, Mortara A, Specchia G. Protective role of chronic statin therapy in reducing myocardial damage during percutaneous coronary intervention. J Cardiovasc Med (Hagerstown). 2006;7(6):416-21. 